\title{
RE: treatment dropout in a family-based partial hospitalization program for eating disorders
}

\author{
Tomoyuki Kawada ${ }^{1}$ iD
}

Received: 28 January 2020 / Accepted: 6 February 2020 / Published online: 17 February 2020

(c) Springer Nature Switzerland AG 2020

I read with great interest the article by Rienecke [1]. The author assessed factors associated with treatment dropout in a partial hospitalization program for adolescents and young adults with anorexia nervosa (AN). Parental criticism was a potentially modifiable factor for avoiding treatment dropout. I have two concerns about the study.

First, Dobrescu et al. conducted a case-control long-term survey to investigate the outcomes in subjects with adolescent-onset AN [2]. They handled 51 cases and the same number of controls. $19 \%$ had an eating disorder diagnosis, $38 \%$ had other psychiatric diagnoses, and $64 \%$ had full eating disorder symptom recovery, which was defined as being free of all eating disorder criteria for 6 consecutive months. The mean duration of an eating disorder was 10 years and $23 \%$ did not receive psychiatric treatment. In addition, later age at onset was selected as a predictor of a good outcome. About one-fourth of the subjects did not receive psychiatric treatment and I suspect that the authors collected subjects with treatment dropout and no history of treatment. Caution should be paid because they observed no events of death during the follow-up and selection bias definitely existed. Besides, the severity of AN and the contents of treatment were not presented, which might be related to the prognosis of AN. Anyway, information on treatment continuity and dropout are supposed to be closely associated with the prognosis of AN.

Second, the authors previously examined the relation between parental expressed emotion (EE) and treatment outcome among adolescents with AN [3]. They observed that paternal criticism predicted lesser improvement at the end of treatment. In addition, the authors recognized that maternal and paternal EE might differentially impact treatment outcome. Furthermore, Uehara et al. reported that

Tomoyuki Kawada

kawada@nms.ac.jp

1 Department of Hygiene and Public Health, Nippon Medical School, 1-1-5 Sendagi, Bunkyo-Ku, Tokyo 113-8602, Japan psychoeducation by EE for the family members of patients might lead to lower distress and encourage positive interactions within the family [4]. Further analysis is recommended to explore factors relating to treatment dropout with particular reference to $\mathrm{EE}$.

Funding None.

\section{Compliance with ethical standards}

Conflict of interest The author declares that there are no conflicts of interest.

Ethical approval This article contains no studies with human participants or animals performed by the author.

Informed consent For this type of study, formal consent is not required.

\section{References}

1. Rienecke RD (2019) Treatment dropout in a family-based partial hospitalization program for eating disorders. Eat Weight Disord 24(1):163-168. https://doi.org/10.1007/s40519-018-0543-9

2. Dobrescu SR, Dinkler L, Gillberg C et al (2019) Anorexia nervosa: 30-year outcome. Br J Psychiatry. https://doi.org/10.1192/ bjp. 2019.113

3. Rienecke RD, Accurso EC, Lock J et al (2016) Expressed emotion, family functioning, and treatment outcome for adolescents with anorexia nervosa. Eur Eat Disord Rev 24:43-51. https://doi. org/10.1002/erv.2389

4. Uehara T, Kawashima Y, Goto M et al (2001) Psychoeducation for the families of patients with eating disorders and changes in expressed emotion: a preliminary study. Compr Psychiatr 42:132138. https://doi.org/10.1053/comp.2001.21215

Publisher's Note Springer Nature remains neutral with regard to jurisdictional claims in published maps and institutional affiliations. 